\title{
Acute Effects of Tumor Necrosis Factor on the Microcirculation in Rat Cremaster Muscle
}

\author{
Eric Vicaut,"* Xin Hou," Didier Payen," Anne Bousseau," and Alain Tedgui" \\ ${ }^{*}$ Laboratoire de Biophysique, Hopital F. Widal, Paris; ${ }^{\ddagger}$ Institut National de la Santé et de la Recherche Médicale, Unité 141 , \\ Hopital Lariboisière, Paris; 'Departement d'anesthésie, Hopital Lariboisière, Paris; and "Rhone Poulenc Santé, Centre \\ de Recherche, Vitry, France
}

\begin{abstract}
The acute effects of TNF on the microcirculation were studied by in vivo microscopy in rat cremaster muscle. The changes in arteriolar diameter after topical administration of recombinant TNF (rTNF; $10^{-4}-10^{4} \mathrm{ng} / \mathrm{ml}$ ) were studied in second-, third-, and fourth-order arterioles (A2-A4) whose mean diameters under control conditions were $64.3,30.7$, and $14.8 \mu \mathrm{m}$, respectively. rTNF induced a concentration-dependent vasodilation whose amplitude was largest for the smallest arterioles. At the highest concentration tested, arteriolar diameter increased by 21,29 , and $41 \%$ of control diameter for the $A 2, A 3$, and $A 4$ arterioles, respectively. Indomethacin or mefenamic acid, two structurally different prostaglandin synthesis inhibitors, markedly inhibited the degree of vasodilation induced by rTNF in the three arteriolar orders. As regards the effect of rTNF on vasoconstriction in response to norepinephrine, vasoconstriction was greatest for the smallest arterioles, and did not change 10 min after rTNF administration for any of the three arteriolar orders. We conclude that $(a)$ rTNF has a direct vasodilatory effect which is greatest in the smallest arterioles, $(b)$ this vasodilation is at least partly mediated by prostaglandins, and (c) administration of rTNF in itself does not acutely alter the response of the arterioles to vasopressive drugs. (J. Clin. Invest. 1991.87:1537-1540.) Key words: tumor necrosis factor • sepsis - microcirculation
\end{abstract}

\section{Introduction}

Cachectin/tumor necrosis factor-alpha (TNF) has been shown to possess tumoricidal activity, which makes it a promising agent in anticancer treatment. Unfortunately, in addition to its beneficial effect, TNF has been found to be involved in the pathological effects provoked by gram-negative bacteria or endotoxin lipopolysaccharides in animals (1-3) and humans (4). Injection of recombinant human TNF (rTNF) ${ }^{1}$ into healthy animals has been found to induce shock with hemodynamic, hormonal, biological, and tissue injury patterns similar to those observed in septic shock $(3,5-7)$. In addition, pretreatment of baboons with anti-TNF monoclonal antibodies pre-

Address reprint requests to Dr. E. Vicaut, Laboratoire de Biophysique, Hopital F. Widal, 200 rue du Fg. St.-Denis, 75010 Paris, France.

Received for publication 6 September 1990 and in revised form 16 November 1990.

1. Abbreviations used in this paper: $\mathrm{rTNF}$, recombinant human tumor necrosis factor.

J. Clin. Invest.

(c) The American Society for Clinical Investigation, Inc.

$0021-9738 / 91 / 05 / 1537 / 04 \$ 2.00$

Volume 87, May 1991, 1537-1540 vented shock after infusion of a lethal dose of Escherichia coli (8).

In septic shock, vasoplegia with acute circulatory collapse requiring the infusion of very high doses of vasoactive drugs is one of the most critical events for prognosis. An important aspect of these hemodynamic disorders concerns the changes occurring in the microcirculatory network, where several mechanisms of pressure regulation are located. Recent studies have shown that septic shock induced by endotoxin or E. coli may affect arteriolar diameter by constricting large arterioles and dilating small terminal arterioles $(9,10)$. However, it is not known whether TNF is involved in these morphological alterations of the microcirculatory network. This study was therefore carried out to quantify the acute response of the arterioles to rTNF per se and to establish whether or not this response was dependent on arteriolar diameter.

Certain findings have shown some degree of interaction between prostaglandin synthesis and responses to TNF. Thus, high levels of plasma prostaglandins were found during sepsis (11), administration of TNF has been shown to induce prostacyclin synthesis in cultured vascular cells (12), and cyclooxygenase inhibitors have been found to reduce TNF toxicity (1315). To test the hypothesis that the acute effect of rTNF on the microcirculation is partly mediated by prostaglandins, we studied the arteriolar responses to rTNF administration in the presence of indomethacin or mefenamic acid, two structurally different prostaglandin synthesis inhibitors. Lastly, as septic shock is associated clinically with reduced sensitivity to vasopressive drugs, we also examined the acute effects of rTNF on the response of arterioles to norepinephrine.

\section{Methods}

Male Sprague-Dawley rats weighing $220 \pm 4 \mathrm{~g}$ (mean \pm SEM) were anesthetized by intraperitoneal injection of $50 \mathrm{mg} / \mathrm{kg}$ sodium pentobarbital. A patent airway was maintained with a tracheotomy tube. The carotid artery was cannulated for measurement of systemic mean arterial blood pressure with a Statham P23DB transducer. All animals whose mean pressure fell to $<90 \mathrm{mmHg}$ were excluded.

\section{Preparation of the cremaster}

After anesthesia, the right cremaster muscle was surgically prepared for in vivo visualization by a new technique described in detail elsewhere (16). Briefly, the muscle was detached from the scrotum but was not longitudinally incised as in the original procedure. A transverse buttonhole slit about 5-mm long was made in the proximal part of the cremaster pouch. The testicle and epididymis, and the cremaster itself were then drawn out through the button-hole. This procedure led to the invagination of the cremaster, which acquired a finger shape, with the cremaster pouch now turned inside out. The small pedicle which attaches the cremaster to the testicle was ligated with two stitches and cut between them, so as to separate the cremaster completely from the testicle, which was reincorporated into the abdominal cavity. To pre- 
pare the cremaster muscle for transillumination microscopy, a flexible extendible ovoid ring was made with metal wire (diameter, $0.1 \mathrm{~mm}$ ) covered with silastic rubber. The ring was then secured in its axis with a clamp and introduced longitudinally into the cremaster pouch. When the clamp was removed the ring expanded gently, spreading out the cremaster which acquired a racket shape. The ring had been positioned so that the main cremaster artery was in the center of the racket's upper surface. Throughout these procedures, the muscle was continuously bathed with warm saline solution.

This new preparation procedure involves minimal incision of the cremaster and consequently reduces considerably the risk of hemorrhage and of lesions to the muscle and its microcirculation. Because the size of the ring is adapted to the dimensions of the cremaster, the extension of the muscle is sufficient to allow good optical resolution, but does not affect the microcirculation. We verified in previous studies that the blood flows observed for each type of arteriole were in the range of those measured with the preparation of Baez (17) which is the one most often used for in vivo microscopy in cremaster muscle.

The muscle chamber was filled with a modified Krebs-Henseleit solution containing, in millimolar per liter: $118 \mathrm{NaCl}, 5.9 \mathrm{KCl}, 2.5$ $\mathrm{CaCl}_{2} \cdot 2 \mathrm{H}_{2} \mathrm{O}, 0.5 \mathrm{MgSO}_{4} \cdot 7 \mathrm{H}_{2} \mathrm{O}, 28 \mathrm{NaHCO}_{3}$, and 10 glucose. This solution was thermostated to ensure a temperature of $34.5^{\circ} \mathrm{C}$ above the cremaster. By bubbling the solution with a $6 \% \mathrm{CO}_{2}-94 \% \mathrm{~N}_{2}$ gas mixture, we fixed the $\mathrm{pH}, \mathrm{PO}_{2}$, and $\mathrm{PCO}_{2}$ of this solution in the muscle chamber at $7.41 \pm 0.04,23 \pm 1.3$, and $42 \pm 0.8 \mathrm{mmHg}$, respectively. The chamber was covered with a plexiglass plate to isolate it from the atmosphere.

To visualize the microcirculation, the chamber was placed on the movable stage of a modified Leitz microscope and the cremaster muscle was transilluminated using a $100-\mathrm{W}$ tungsten-halogen lamp. The image, magnified by a $20 \times$ objective and $10 \times$ oculars, was projected into a CCD camera (Sony) connected to a professional videotape recorder (Sony VO 9600 P). Arteriolar diameters were measured by playback analysis of the video record using the technique of Intaglietta (18) and a distance measurement device (IPM 303 dimension analyzer, San Diego, CA). Depending on the geometry of the part of the network studied, two to four arterioles were examined in each experiment.

\section{Experimental protocol}

Determination of the effects of rTNF on the arteriolar network as a function of concentration and arteriolar order. In nine rats, dose-response relationships were established by measuring the effects on arteriolar diameter of increasing the concentration of rTNF in the suffusion bath. The studied arterioles were randomly selected and their basal tone was verified by checking their ability to dilate in the presence of $10^{-4}$ adenosine. Arteriolar orders were identified by their relative locations in the network according to morphological criteria derived from Zweifach and Lipowsky (19). After washing out the adenosine and return to control conditions, control diameter was measured and rTNF was then added to the bath at increasing concentrations. In all experiments we considered the maximal degree of vasodilation, which was always reached in $<5 \mathrm{~min}$.

Recombinant human TNF- $\alpha$ was derived from $E$. coli. Purified recombinant molecules were $>95 \%$ homogeneous and endotoxin content was $<5 \mathrm{ng} / \mathrm{mg}$. The activity of rTNF, based on its cytotoxicity towards $\mathrm{L} 929$ cells, was $3 \times 10^{6} \mathrm{U} / \mathrm{mg}$. All concentrations referred to final bath concentrations.

To exclude the possibility that contamination of the rTNF used here might affect the results, we studied, in four rats, the effect of administering a sample of $r-T N F$ that had been preincubated for $30 \mathrm{~min}$ with rabbit serum anti-human TNF (Genzyme Corp., Boston, MA). The concentration of TNF used for this test was 10 times higher than the highest concentration studied in the concentration-response curve.

Determination of the effect of $r$ TNF in the arteriolar network in the presence of prostaglandin synthesis inhibitors. In 12 rats, we studied the dilatory effect of $10^{2} \mathrm{ng} / \mathrm{ml}$ rTNF in the presence of inhibitors of cyclooxygenase. To avoid possible nonspecific effects, separate studies were conducted using two structurally dissimilar inhibitors: indomethacin $\left(2.8 \times 10^{-5} \mathrm{M}\right)$ and mefenamic acid $\left(1.9 \times 10^{-5} \mathrm{M}\right)$, which were added to the bath 10 min before rTNF administration. Because administration of cyclooxygenase inhibitors may reduce basal diameter, vasodilations were expressed as a function of the diameter of the arterioles immediately before TNF administration. The degree of vasodilation obtained for each arteriolar order was compared to that obtained in protocol A for the same order at the same concentration of rTNF. Indomethacin and mefenamic acid (Sigma Chemical Co., St. Louis, MO) were dissolved in $1 \mathrm{mg} / \mathrm{ml}$ sodium carbonate and then diluted to final concentrations with the Krebs buffer used for the cremaster bath. All drugs were prepared fresh daily. Control studies excluded any effect of the vehicles.

Arteriolar response to norepinephrine after rTNF administration. In 12 rats, the response to topical administration of $10^{-5} \mathrm{mg} / \mathrm{ml}$ norepinephrine ( $d$-bitartrate from Winthrop dissolved in Krebs buffer) was measured and considered as the control constriction for the studied arterioles. The bath was then drained and the muscle carefully washed several times and exposed to $10^{2} \mathrm{ng} / \mathrm{ml}$ rTNF. The same arterioles as those initially exposed to norepinephrine were again exposed to the same dose, and the resulting constriction was compared to the control degree of constriction. In preliminary experiments, we verified that the response of an arteriole to the second exposure to norepinephrine was not different from its response to the first.

\section{Statistical analysis}

Log (dose)-response relationships were analyzed using a two-way analysis of variance for repeated measures and decomposition of the factor "dose" into orthogonal polynomials to test differences between the response curves of the three arteriole orders (20). The responses observed in protocols $B$ and $C$ were respectively analyzed using two-way analysis of variance for independent and repeated measures. The level of significance was fixed at $5 \%$.

All tests were performed using Biomedical Data Package statistical software (University of California at Los Angeles).

\section{Results}

Mean blood pressure was $109 \pm 4 \mathrm{mmHg}$. No significant changes were observed during the experiments.

Results were expressed as a function of the arteriolar order. Mean control diameters $( \pm \mathrm{SEM})$ were respectively $64.3 \pm 4.1$, $30.7 \pm 1.6$, and $14.8 \pm 1.3 \mu \mathrm{m}$ for the second, third, and fourth arteriole orders (A2, A3, and A4). For each order, the control diameters of the arterioles were not different in the three parts of the protocol.

Effect of rTNF on the arteriolar diameter as a function of concentration and arteriolar order. At concentrations of $10^{-2}$ and $10^{4} \mathrm{ng} / \mathrm{ml}$, rTNF was found to have a significant dose-dependent vasodilatory effect on the three arteriolar orders studied $(P<0.001$; Fig. 1). Comparisons of the slopes of the $\log$ (dose)-response curves showed that A4 arterioles $(n=6)$ were more sensitive to rTNF than A2 $(n=5)$ or A3 arterioles $(n=7)$ $(P<0.05)$. No significant difference was found between the slopes for $\mathrm{A} 2$ and $\mathrm{A} 3$ arterioles.

No significant differences in diameter were found, when $10^{5} \mathrm{ng} / \mathrm{ml} \mathrm{rTNF}$ preincubated with TNF antiserum, was applied on the microcirculation because diameters for the $\mathrm{A} 2$, A3, and A4 orders ( $n=5$ for all three) were respectively $63.0 \pm 3.5$ vs. $62.5 \pm 4.0,26.4 \pm 6.1$ vs. $25.8 \pm 6.5$, and $13.4 \pm 1.15$ vs. $13.7 \pm 1.15$.

Effect of $r T N F$ on the arteriolar diameter in the presence of prostaglandin synthesis inhibitors. The addition of $2.8 \times 10^{-5}$ $M$ indomethacin to the cremaster muscle resulted in a slight but significant decrease in basal diameters, which reached $94 \pm 2,90 \pm 5$, and $91 \pm 4 \%$ of the basal values for the A2 $(n=5)$, A3 $(n=6)$, and A4 $(n=7)$ orders, respectively $(P<0.05)$. The responses to $10^{2} \mathrm{ng} / \mathrm{ml}$ rTNF in the presence of indomethacin 


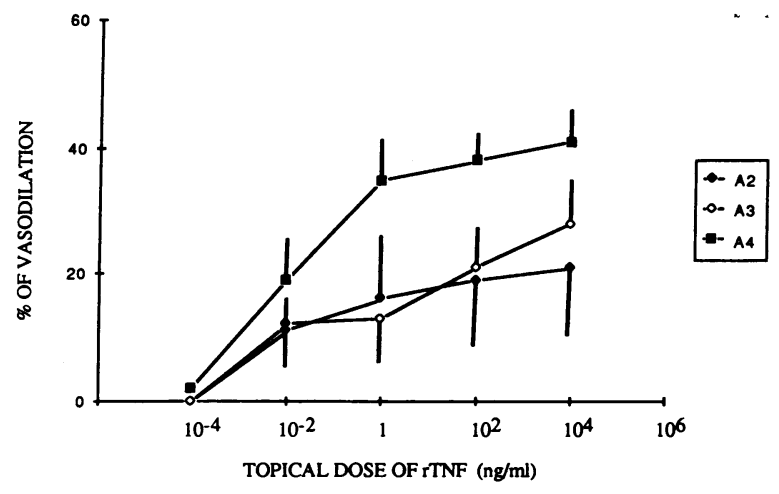

Figure 1. Dose-response curves (percentage of vadilation $[ \pm \mathrm{SEM}]$ vs. $\log$ [dose]) for response by second- to fourth-order arterioles (A2-A4) to topical administration of rTNF.

were compared with the response of the corresponding arteriolar order to the same dose of rTNF only. With indomethacin, the degree of rTNF-dependent dilation dropped significantly for all three arteriolar orders $(P<0.05$; Fig. 2$)$. No significant interaction was found between the factor "arteriolar order" and the factor "presence or absence of indomethacin" showing that the inhibition was not significantly different for the three arteriolar orders studied. The results obtained with $1.9 \times 10^{-5}$ M mefenamic acid were similar to those for indomethacin (Fig. 2), because mefenamic acid also reduced diameters to $95 \pm 2$, $87 \pm 5$, and $91 \pm 4 \%$ of the basal values for the A2 $(n=5), \mathrm{A} 3(n$ $=6)$, and A4 $(n=6)$ orders, respectively. Like indomethacin, mefenamic acid also significantly reduced rTNF-dependent dilation $(P<0.01)$. This reduction was not significantly different for the three arteriolar orders. Finally, the responses observed with mefenamic acid were not significantly different from the responses found with indomethacin.

Arteriolar response to norepinephrine after $r T N F$ administration. As shown in Fig. 3, the responses to norepinephrine after administration of rTNF in A2 $(n=9), \mathrm{A} 3(n=9)$, and A4 arterioles $(n=6)$ did not change significantly in any of the three arteriolar orders.

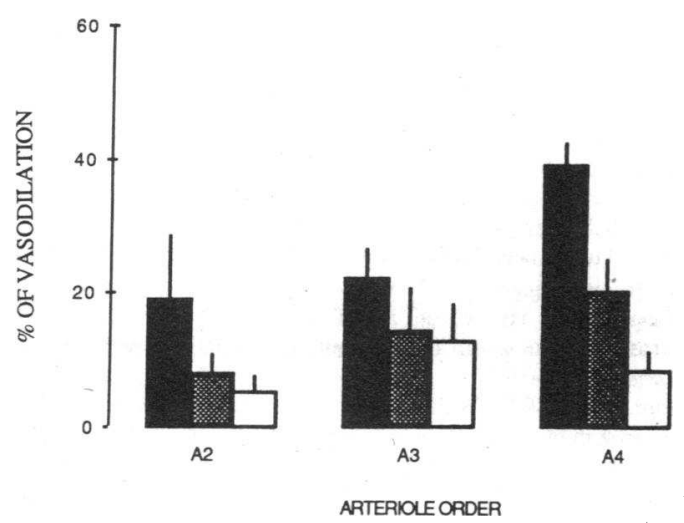

Figure 2. Comparisons of the vasodilatory responses to $10^{2} \mathrm{ng} / \mathrm{ml}$ rTNF (mean \pm SEM) by second- to fourth-order arterioles (A2-A4) with pretreatment by $2.8 \times 10^{-5} \mathrm{M}$ indomethacin (stippled bars), or $1.910^{-5} \mathrm{M}$ mefenamic acid (open bars), or without pretreatment (solid columns). For the three arteriolar orders studied, the responses to rTNF + indomethacin or mefenamic acid were significantly different from those to rTNF alone. The arteriolar order did not signifcantly affect the amplitude of this difference. No significant differences were found between the groups respectively pretreated by indomethacin and mefenamic acid.

\section{Discussion}

Several studies have shown that TNF plays a major role in the pathogenesis of septic shock. However, recent reports indicated some degree of synergy between TNF and other cytokines (15) and between TNF and bacteria or their products (21). Consequently, its direct implication in the hemodynamic events associated with sepsis remains to be elucidated.

Recently, Cryer et al. $(9,10)$ found that during septic shock in the rat, large arterioles constricted and small terminal arterioles dilated. These studies showed that changes in the microvascular network might be responsible for some of the hemodynamic alterations found in sepsis, but did not provide any information about the potential role of TNF in these morphological alterations. The present study showed that in the absence of shock, TNF in itself induced vasodilation in the arteriolar network, for all the three arteriolar orders studied. The arteriolar responses observed were certainly not due to the reaction of microcirculatory regulatory mechanisms to systemic hemodynamic changes, because rTNF was administered topically and no changes in systemic blood pressure were observed. As shown by the dose-response relationships, the range of TNF concentrations inducing vasodilation included those at which TNF was found to interact with endothelial cells in vitro (22) and those at which it was present during septic shock in rabbits (2), baboons (8), and humans $(4,23)$. As shown in Fig. 1 , the sensitivity of the arterioles to rTNF was dependent on their order, as the largest ones dilated about half as much as the smallest at the highest concentration tested. This observation was in accordance with many other reports showing various responses to vasoactive substances in different parts of the arteriolar network. The vasodilation gradient observed here might be due to the differences in wall stress in this network, which interfere with vasoactivity (24), or to differences in TNF receptors. An additional explanation might be local differences in the characteristics of receptors for vasodilating substances, whose release might be TNF-dependent.

The maximal individual vessel responses observed with TNF were found to be between 30 and $80 \%$ of those found in the same arterioles after topical application of $1 \times 10^{-4} \mathrm{M}$ adenosine. With high concentrations of TNF the average vasodilations observed (i.e., 21,29 , and $41 \%$ for $\mathrm{A} 2$, A3, and $\mathrm{A} 4$,

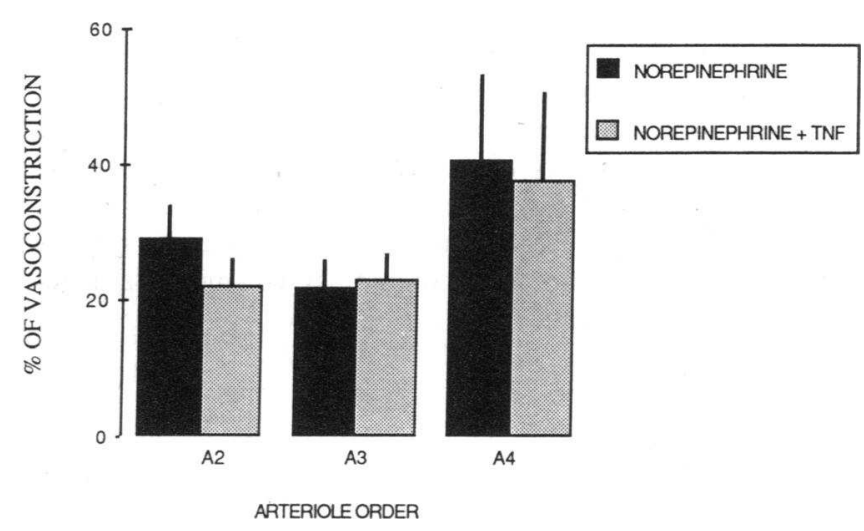

Figure 3. Comparison of the vasoconstrictive responses to topical administration of $10^{5} \mathrm{mg} / \mathrm{ml}$ norepinephrine (mean $\pm S E M$ ) by secondto fourth-order arterioles (A2-A4, solid bars) with their responses to the same dose of norepinephrine administered $10 \mathrm{~min}$ after exposure to $10^{2} \mathrm{ng} / \mathrm{ml}$ rTNF (stippled bars). No significant differences were found for the three arteriolar orders studied. 
respectively) were also smaller than those reported by others in cremaster microvessels after topical administration of $1 \times 10^{-4}$ $M$ adenosine (i.e., 30,68 , and $100 \%$ for diameters corresponding to A2, A3 [25], and A4 arterioles [26]). Because this concentration of adenosine is considered to produce maximal dilation of microvessels, it is clear that TNF cannot be considered to induce maximal dilation of the skeletal muscle microvascular network. However, as arterioles are one of major sites of pressure regulation, and vascular hindrance is inversely proportional to the fourth power of the vessel diameter, the $20-40 \%$ vasodilation induced by TNF may account for a significant portion of the decrease in blood pressure observed in septic shock.

An increase in the basal tone of arterioles after cyclooxygenase inhibition was found by others in in vivo studies of skeletal muscle microcirculation (27), and was due to the role of eicosanoids in regulating microvascular tone. Here, the marked inhibition of vasodilation by cyclooxygenase inhibitors indicated that the vasodilation induced by TNF was partly mediated by an additional production or release of prostaglandins. This finding is in accordance with the high level of plasma prostaglandins reported in sepsis (11) and after TNF administration in endothelial cell cultures (12). It also agrees with the hypothesis that prostaglandins may mediate some of the hemodynamic disorders that occur in sepsis (11). The weaker microvascular responses to rTNF observed with prostaglandin synthesis inhibitors might also play a role in the reduction of TNF toxicity, reported with these drugs (13-15). Very recently, overproduction of nitric oxide was found to be associated with TNF administration (28). Acute local overproduction of nitric oxide at the microvascular level might account for that part of the vasodilation which persisted here in the presence of cyclooxygenase inhibitors, a possibility which requires further study.

Despite clinical evidence of reduced sensitivity to vasopressive drugs in patients with septic shock, we did not find any decrease in the response of cremaster muscle arterioles to norepinephrine after rTNF administration. This suggests that the vascular hyporesponsiveness to contraction agonists observed in septic shock is not due to an acute effect of TNF. Nevertheless, the present results do not allow us to conclude that this reduced responsiveness is due to mechanisms independent of TNF or to mechanisms which are initiated by TNF but require a longer period than the present observation time or synergy with other products to be effective.

In summary, we found that acute topical administration of rTNF had a vasodilatory effect on the arteriolar network. Its amplitude was largest for the smallest arterioles. Vasodilation induced by rTNF was greatly reduced by prostaglandin synthesis inhibitors and was not found to interact with arteriolar reactivity to norepinephrine.

\section{References}

1. Beutler, B. A., I. W. Milsark, and A. C. Cerami. 1985. Passive immunization against cachectin/tumor necrosis factor protects mice from lethal effect of endotoxin. Science (Wash. DC). 229:869-871.

2. Beutler, B. A., I. W. Milsark, and A. C. Cerami. 1985. Cachectin/tumor necrosis factor: production, distribution, and metabolic fate in vivo. J. Immunol. 135:3972-3977.

3. Tracey, K. J., B. Beutler, S. F. Lowry, J. Merryweather, S. Wolpe, I. W.
Milsark, R. J. Hariri, T. J. Fahey, A. Zentella, J. D. Albert, G. T. Shires, and A. Cerami. 1986. Shock and tissue injury induced by recombinant human cachectin. Science (Wash. DC). 234:470-474.

4. Michie, H. R., K. R. Manogue, D. R. Spriggs, A. Revhaug, S. O'Dwyer, C. A. Dinarello, A. Cerami, S. M. Wolff, and D. W. Wilmore. 1988. Detection of circulating tumor necrosis factor after endotoxin administration. N. Engl. J. Med. 318:1481-1486.

5. Bauss, F., W. Dröge, and D. N. Männel. 1987. Tumor necrosis factc mediates endotoxic effects in mice. Infect. Immun. 55:1622-1625.

6. Tracey, K. J., S. F. Lowry, T. J. Fahey, J. D. Albert, Y. Fong, D. Hesse, B. Beutler, K. R. Manogue, S. Calvano, H. Wei, and A. Cerami. 1987. Cachectin/tumor necrosis factor induces lethal shock and stress hormones in the dog. Surg. Gynecol. Obstet. 164:415-424.

7. Remick, D. G., R. G. Kunkel, J. W. Larrick, and S. L. Kunkel. 1987. Acute in vivo effects of human recombinant tumor necrosis factor. Lab. Invest. 56:583590.

8. Tracey, K. J., Y. Fong, D. G. Hesse, K. R. Manogue, A. T. Lee, G. C. Kuo, S. F. Lowry, and A. Cerami. 1987. Anti-cachectin/TNF monoclonal antibodies prevent septic shock during lethal bacteremia. Nature (Lond.). 330:662-664.

9. Cryer, H. M., R. N. Garrison, H. W. Kaebnick, P. D. Harris, and L. M. Flint. 1987. Skeletal microcirculatory responses to hyperdynamic Escherichia coli sepsis in unanesthetized rats. Arch. Surg. 122:86-92.

10. Cryer, H. M., R. N. Garrison, and P. D. Harris. 1988. Role of muscle microvasculature during hyperdynamic and hypodynamic phases of endotoxin shock in decerebrate rats. J. Trauma. 28:312-318.

11. Fletcher, J. R. 1982. The role of prostaglandins in sepsis. Scand. J. Infect. Dis. 31(Suppl.):55-60.

12. Kawakami, M., S. Ishibashi, H. Ogawa, T. Murase, F. Takaku, and S. Shibata. 1986. Cachetin/TNF as well as interleukin-1 induces prostacyclin synthesis in cultured vascular endothelial cells. Biochem. Biophys. Res. Commun. 141:482-487.

13. Evans, D. A., D. O. Jacobs, A. Revhaug, and D. W. Wilmore. 1989. The effects of tumor necrosis factor and their selective inhibition by ibuprofen. Ann. Surg. 209:312-321.

14. Kettelhut, I. C., W. Fiers, and A. L. Goldberg. 1987. The toxic effects of tumor necrosis factor in vivo and their prevention by cyclooxygenase inhibitors. Proc. Natl. Acad. Sci. USA. 84:4273-4277.

15. Okusawa, S., J. A. Gelfand, T. Ikejima, R. J. Connolly, and C. A. Dinarello. 1988. Interleukin 1 induces a shock-like state in rabbits: synergism with tumor necrosis factor and the effect of cyclooxygenase inhibition. J. Clin. Invest. 81:1162-1172.

16. Vicaut, E., G. Montalescot, X. Hou, O. Stücker, and B. Teisseire. 1989. Arteriolar vasoconstriction and tachyphylaxis with intraarterial angiotensin II. Microvasc. Res. 37:28-41.

17. Baez, S. 1973. An open cremaster preparation for the study of blood vessels by in vivo microscopy. Microvasc. Res. 5:384-394.

18. Intaglietta, M., and W. R. Tompkins. 1973. Microvascular measurements by video image shearing and splitting. Microvasc. Res. 5:309-312.

19. Zweifach, B. W., and H. H. Lipowsky. 1984. Pressure-flow relations in blood and lymph microcirculation. In Handbook of Physiology. Vol. IV. The Cardiovascular System. E. Renkin and C. C. Michel, editors. American Physiology Society, Bethesda, MD. 251-305.

20. Winer, B. J. 1971. Statistical Principles in Experimental Design. McGrawHill Book Co., New York.

21. Rothstein, J. L., and H. Scfreiber. 1988. Synergy between tumor necrosis factor and bacterial products causes hemorrhagic necrosis and lethal shock in normal mice. Proc. Natl. Acad. Sci. USA. 85:607-611.

22. Frater-Schröder, M. F., W. Risau, R. Hallmann, P. Gautschi, and P. Böhlen. 1987. Tumor necrosis factor type a, a potent inhibitor of endothelial cell growth in vitro, is angiogenic in vivo. Proc. Natl. Acad. Sci. USA. 84:5277-5281.

23. Waage, A., A. Halstensen, and T. Espevik. 1987. Association between tumor necrosis factor in serum and fatal outcome in patients with meningococcal disease. Lancet. i:355-357.

24. Gore, R. W. 1972. Wall stress: a determinant of regional differences in response of frog microvessels to norepinephrine. Am. J. Physiol. 222:82-91.

25. Meininger, G. A., K. L. Fehr, and M. B. Yates. 1985. Anatomic and hemodynamic characteristics of the blood vessels feeding the cremaster skeletal muscle in the rat. Microvasc. Res. 33:81-97.

26. Koller, A., E. J. Messina, M. S. Wolin, and G. Kaley. 1989. Effects of endothelial impairment on arteriolar dilation in vivo. Am. J. Physiol. 257:H1485-H1489.

27. Koller, A., E. J. Messina, M. S. Wolin, and G. Kaley. 1989. Endothelial impairment inhibits prostaglandin and EDRF-mediated arteriolar dilation in vivo. Am. J. Physiol. 257:H1966-H1970.

28. Kilbourn, R. G., S. S. Gross, A. Jubran, J. Adams, O. W. Griffith, R. Levi, and R. F. Lodato. 1990. $\mathrm{N}^{\mathrm{G}}$-Methyl-L-arginine inhibits tumor necrosis factor-induced hypotension: implications for the involvement of nitric oxide. Proc. Natl. Acad. Sci. USA. 87:3629-3632. 